\title{
Editorial
}

\section{The National Cancer Survivorship Initiative: new and emerging evidence on the ongoing needs of cancer survivors}

\author{
M Richards*, , J Corner ${ }^{2,3}$ and J Maher ${ }^{3}$ \\ 'National Cancer Action Team, 18th Floor Portland House, Bressenden Place, London SWIE 5RS, UK; ${ }^{2}$ Faculty of Health Sciences, University of \\ Southampton, Building 67, Highfield Campus, Southampton SOI 7 I BJ, UK: ${ }^{3}$ Macmillan Cancer Support, 89 Albert Embankment, London SEI 7 UQ, UK
}

British Journal of Cancer (20II) 105, SI -S4; doi: 10.1038/bjc.20II.4I6 www.bjcancer.com

(c) 20II Cancer Research UK

A key commitment of the Cancer Reform Strategy (Department of Health, 2007) in UK, and endorsed in Improving Outcomes Strategy for Cancer (Department of Health, 2011), was to establish a National Cancer Survivorship Initiative (NCSI). The initiative was launched in January 2010 and is co-led by the Department of Health and Macmillan Cancer Support with the involvement of a large range of stakeholders. The Improving Outcomes Strategy for Cancer recognises that not enough attention has been given to the long-term consequences of a cancer diagnosis for the ever increasing number of individuals surviving the disease, or as to how to enable individuals to return to active lives following the completion of initial cancer treatment. The Survivorship Initiative has set out to understand the needs of those living with cancer and develop models of care that meet their needs, the goal being to support cancer survivors to live as healthy and active a life as possible, for as long as possible. The NCSI sets a vision based on five shifts in care and support for people living with and beyond cancer (Figure 1).

A core focus for the NCSI has been to engage members of the research community to review and develop the evidence in relation to the needs of cancer survivors and the most effective models of services, care and support to address these. A major issue for the Initiative has been the lack of UK data and evidence relating to the health outcomes (other than survival) for individuals following primary treatment for cancer. The need to stimulate a new field of enquiry to address gaps in data and evidence has been recognised. The aim of this supplement to the British Journal of Cancer is to bring together new and emergent research in the field of cancer survivorship.

\section{CANCER SURVIVORSHIP: A NEW FIELD OF ENQUIRY}

It is both a tribute to the success of cancer research and treatment over the last 40 years and an indictment of these efforts that we need a National Cancer Survivorship Initiative. Over the period, longterm survival for individuals diagnosed with cancer has doubled and there is now a life expectancy of 10 years or more for the majority of individuals with some common cancers (although there are notable exceptions in lung and pancreatic cancers where survival rates remain stubbornly poor; CRUK, 2010). These developments are transforming the experience of cancer from one where in the past the disease inevitably signalled a potentially life-threatening illness

\footnotetext{
*Correspondence: Professor, Sir M Richards;
}

E-mail: Mike.richards@ncat.nhs.uk to one where today we are beginning to think of cancer in certain circumstances as a long-term, even a 'chronic' illness (Tritter and Calnan, 2002). Nevertheless, in 2007, when work for the Survivorship Initiative began, we had no recent epidemiological data relating to the number of individuals living with a cancer diagnosis in the UK; less importance was attributed to measuring prevalence than to incidence, survival and mortality as core measures of cancer outcomes, and there were no data relating to broader dimensions of health outcomes among cancer survivors. Since 2007, work has commenced to fill in these gaps and the results of these efforts are presented in a number of papers in this supplement. Evidence from this early work indicates concerns over the short- and long-term health outcomes for individuals following initial cancer treatment and suggests that great attention needs to be paid to this as part of routine cancer management. However, there has been insufficient data available to provide detailed evidence as to how best to address these concerns or the specific actions that should be taken to improve services or care.

If health outcomes for cancer survivors are to be improved, we need answers to the following questions:

1. How many people are currently living with a cancer diagnosis, and how is this likely to change over time?

2. What is the state of health and well-being of cancer survivors, and how does this compare with that of people with or without other long-term conditions?

3. What specific problems, concerns or needs do cancer survivors report at different times after diagnosis and at different phases in the pathway of care?

4. What are the risks of survivors experiencing adverse consequences from cancer treatment at different time intervals after diagnosis?

5. What care are cancer survivors currently receiving from the NHS in hospitals and in the community?

6. How do cancer survivors perceive the care they currently receive and what are their preferences for future care?

7. What interventions have been shown to improve health outcomes for cancer survivors?

8. How can survivorship care best be delivered?

9. What do we not know that should form priorities for future research?

10. How can research in this important field best be supported?

The papers included in this supplement start to address these questions, particularly from a UK perspective. 
Five shifts in care and support for people living with and beyond cancer:

1. A cultural shift in the approach to care and support for people affected by cancerto a greater focus on recovery, health and well-being after cancer treatment.

2. A shift towards holistic assessment, information provision and personalised care planning. This is a shift from a one-size fits all approach to follow-up to personalised care planning based on assessment of individual risks, needs and preferences.

3. A shift towards support for self-management. This is a shift from a clinically led approach to follow-up care to supported self-management, based on individual needs and preferences. This approach empowers individuals to take on responsibility for their condition supported by the appropriate clinical assessment, support and treatment.

4. A shift from a single model of clinical follow-up to tailored support that enables early recognition of the consequences of treatment and the signs and symptoms of further disease as well as tailored support for those with advanced disease.

5. A shift from an emphasis on measuring clinical activity to a new emphasis on measuring experience and outcomes for cancer survivors through routine use of patient-reported outcome measures in aftercare services.

Figure I The National Cancer Survivorship Initiative for England Vision (Department of Health, Macmillan Cancer Support, NHS Improvement, 20 I0).

\section{NUMBERS OF CANCER SURVIVORS}

Maddams et al (2009) have previously reported a pivotal study indicating that around 2 million people were alive in the UK following a diagnosis of cancer at the end of 2008, and that this figure will rise by $3 \%$ each year. Breast, colorectal and prostate cancer account for a large proportion of all cancer survivors.

In this supplement, Maher and McConnell (2011) build on the overall prevalence figures for breast, colorectal and lung cancer to estimate the numbers of patients likely to be alive at different phases of the survivorship care pathway. At present, cancer registries in the UK do not routinely collect data on date of recurrence or metastasis, and thus these estimates rely on a number of clinically led assumptions. However, the broad estimates should provoke thought and may be helpful for service planners. Collection of data on date of recurrence is now being piloted by breast cancer teams in this country, working with cancer registries. It is anticipated that this work will be extended to other cancers. Refinements of the estimates made by Maher and McConnell should therefore be possible in the future.

\section{HEALTH AND WELL-BEING OF CANCER SURVIVORS}

In 2008, a national population-based survey was undertaken to compare the self-reported health and well-being of individuals with a cancer diagnosis with individuals who had not been treated for cancer, as well as with individuals with other serious chronic or long-term conditions. The survey revealed relatively poorer health and well-being and relatively higher use of hospital services among individuals who had a former cancer diagnosis when compared with individuals who had no cancer diagnosis. Initial data from the survey were reported in the NCSI Vision report (Department of Health, Macmillan Cancer Support, NHS Improvement, 2010). In this Supplement, Elliott et al (2011) report for the first time a detailed secondary analysis of these data and provide evidence that the health profiles of cancer survivors are broadly similar to those with a serious long-term condition. In addition, individuals formerly diagnosed with cancer and who also report a chronic illness are in poorer health still. It is, however, important to note that a significant number of cancer survivors report excellent or good health.

\section{PROBLEMS AND CONCERNS OF CANCER SURVIVORS}

The study reported by Foster and Fenlon (2011) in this supplement highlights a relatively under-recognised problem experienced by a significant number of cancer survivors. This is loss of selfconfidence, which may be a particular issue in the period shortly after completion of primary treatment. Foster and Fenlon (2011) reveal how recovery from primary cancer treatment requires a rebuilding of lost confidence and how cancer survivors may struggle where confidence is low or appropriate support is lacking; indeed, this loss of self-confidence may itself be a significant barrier to accessing support. The authors argue that regaining or recovery of lost self-confidence is an important aspect of recovery alongside physical and psychosocial problems, and that this requires a supportive infrastructure. Programmes that aim to promote rehabilitation and supported self-management should take account of this. Importantly, Foster and Fenlon (2011) have incorporated learning from their research into the design of a study of recovery of health and well-being in a cohort of 1000 colorectal cancer survivors recently launched by the Macmillan Survivorship Research Group at the University of Southampton.

\section{CONSEQUENCES OF TREATMENT}

Khan et al (2011a) have used the General Practice Research Database to explore the incidence of long-term consequences of cancer treatment among 26000 patients diagnosed with breast, colorectal or prostate cancer at least 5 years earlier. Using a casecontrol design, the authors have identified increases in heart failure, coronary heart disease, osteoporosis, diabetes and hypothyroidism among cancer survivors. This study demonstrates the potential of using routinely recorded electronic data from primary care to explore the patterns of ill health experienced by cancer survivors. The use of linked data from primary and secondary care together with information from cancer registries should, in future, facilitate more detailed analyses of risk factors related to specific aspects of anticancer treatment.

\section{HEALTH SERVICE UTILISATION}

Maddams et al (2011a) have built on their previous work on cancer prevalence to assess health service utilisation among cancer 
survivors using linked data from cancer registries and hospital episode statistics. They have previously demonstrated that the overwhelming majority of inpatient admissions among cancer patients occur in the first year after diagnosis or in the year before death (Maddams et al, 2011b). In this supplement, the same authors explore the extent to which these admissions are related or unrelated to the diagnosis of cancer at different periods following diagnosis. Although the majority of hospital activity is in the first year following diagnosis, a significant amount of admissions occur for all cancer survivors in years 1-5, particularly for lung and colorectal cancer, and is indicative of the ongoing needs of cancer survivors. This study also reveals that older cancer survivors (over 75 years) have a higher-level hospitalisation and this is particularly the case for prostate cancer.

\section{PERCEPTIONS AND PREFERENCES FOR CARE}

In parallel with their large-scale quantitative study described above, Khan et al (2011b) have undertaken qualitative interviews to explore perceptions of primary-care services among cancer survivors. Although cancer survivors described ongoing needs for information and support that could potentially be provided by GPs, they reported little involvement by GPs in their care. In addition, GPs were perceived by patients as lacking the necessary expertise in cancer management.

\section{INTERVENTIONS TO ENHANCE HEALTH OUTCOMES}

Davies et al (2011) present a review of the literature on the role of diet and physical activity in breast, colorectal and prostate cancer survivorship. This highlights the importance of physical activity in protecting against cancer recurrence and progression, with a dose-response for better outcomes. When taken with findings from a previous systematic review undertaken by the World Cancer Research Fund (Bekkering et al, 2006), evidence suggests that the mechanism of benefit from diet and physical activity relates to weight, with excess weight being a risk factor for recurrence. The challenge now will be to use this research evidence to promote better outcomes among large numbers of cancer survivors.

\section{SERVICE DELIVERY LEVELS}

The National Cancer Survivorship Initiative is exploring a range of new models of care for patients at different phases of the survivorship pathway. These include new approaches to assessment and care planning, providing advice and support on healthy lifestyles, alternatives to routine follow-up and new models of care for patients with late effects of treatment. To assess the impact of new interventions and service delivery models, it is becoming increasingly important to develop electronic capture of quality-oflife data on cancer survivors. This is already needed for research, but it is likely that this will become a routine part of clinical practice in the future. In order for this to happen, technical platforms are required to collect patient-reported outcome measures on large numbers of patients. In this supplement, Ashley et al (2011) detail the steps being taken to develop and assess one such platform (EPOCS).

\section{PRIORITIES FOR FUTURE SURVIVORSHIP RESEARCH}

A systematic review of current research evidence relating to survivorship was commissioned as part of the NCSI in order to identify priorities for future research. Richardson et al (2011) publish the findings from this review in this supplement. These findings have already informed the development of an action plan for promoting research in this field by the UK National Cancer Research Institute (NCRI, 2010). The highest priority areas for research are identified as follows:

- Delineating the natural history of survivorship

- Creating tools to identify the risk of an individual developing ongoing problems following primary cancer treatment

- Health services research and economic assessment of care pathways for cancer survivors

- Studies investigating the psychological and social impact of cancer

- Studies investigating ongoing physical symptoms experienced by cancer patients as a consequence of cancer treatment

\section{SUPPORT FOR SURVIVORSHIP RESEARCH}

Several years ago the National Cancer Research Institute identified support for research into supportive and palliative care as being a high priority (NCRI, 2004). This led to the establishment of two research collaboratives: CECo (www.ceco.org.uk) and COMPASS (www.compasscollaborative.com). During 2009-2010, the NCRI undertook a rapid review of research in this area (NCRI, 2010) as part of the National Cancer Survivorship Initiative. A key aim of this review was to integrate the strategies of government and charity sector research funders in these areas. The review highlighted the great progress made since 2004, including the expansion of research capacity and the generation of a culture of collaboration of cross-disciplinary and multi-institutional working. Further support to build and sustain research in this area is now being undertaken under the auspices of NCRI.

\section{SUMMARY}

In summary, new models of care are needed to optimise healthrelated quality of life among cancer survivors. The development of these models depends critically on high-quality research. Until recently, this field of cancer research has been relatively underdeveloped both within the UK and elsewhere. There are methodological challenges as the infrastructure for gathering information on long-term health outcomes as part of routinely collected data in the NHS is in the process of being established, and data relating to key problems and measures of these are still emerging. The number of research groups pursuing questions related to cancer survivorship is small at present and needs to increase as does the investment in research that will address the 10 questions highlighted in this editorial. The studies reported in this supplement show that important work is now underway in this country. This is already informing thinking within the NCSI, but many important questions remain unanswered. Further development of research in this field should remain a high priority.

\section{ACKNOWLEDGEMENTS}

We thank all authors who contributed to the supplement and the peer reviewers who provided their time and valued feedback. We are especially grateful to Julie Flynn for coordinating this supplement and to Macmillan Cancer Support for sponsoring it. This article is sponsored by Macmallan Cancer Support. 


\section{REFERENCES}

Ashley L, Jones H, Thomas J, Forman D, Newsham A, Morris E, Johnson O, Velikova G and Wright P (2011) Integrating cancer survivors' experiences into UK cancer registries: design and development of the ePOCS system (electronic Patient-reported Outcomes from Cancer Survivors) Br J Cancer 105(Suppl 1): S74-S81

Bekkering T, Beynon R, Davey Smith G, Davies A, Harbord R, Sterne J, Thomas S, Wood L (2006) A systematic review of RCTs investigating the effect of dietal and physical activity interventions on cancer survival, updated report. World Cancer Research Fund, http://www. dietandcancerreport.org/

Cancer Research UK (2010) http://info.cancerresearchuk.org/news/ archive/pressrelease/2010-07-12-deadly-cancer-survival-doubles

Davies NJ, Batehup L, Thomas R (2011) The role of diet and physical activity in breast, colorectal, and prostate cancer survivorship: a review of the literature. Br J Cancer 105(Suppl 1): S52-S73

Department of Health (2007) Cancer Reform Strategy. http://www.dh. gov.uk/en/publicationsandstatisitics/publications/PublicationsPolicyAnd Guidance/DH_081006

Department of Health (2011) Improving outcomes: a strategy for cancer. http://www.dh.gov.uk/en/Publicationsandstatistics/Publications/Publications PolicyAndGuidance/DH_123371

Department of Health, Macmillan Cancer Support, NHS Improvement (2010) The National Cancer Survivorship Initiative Vision. http://www. dh.gov.uk/en/Publicationsandstatistics/Publications/PublicationsPolicyAnd Guidance/DH_111230

Elliott J, Fallows A, Staetsky L, Smith PWF, Foster CL, Maher EJ, Corner J (2011) The health and well-being of cancer survivors in the UK: findings from a population-based survey. Br J Cancer 105(Suppl 1): $\mathrm{S} 11-\mathrm{S} 20$

Foster C, Fenlon D (2011) Recovery and self-management support following primary cancer treatment. $\mathrm{Br}$ J Cancer 105(Suppl 1): S21 - S28

Khan NF, Evans J, Rose PW (2011b) A qualitative study of unmet needs and interactions with primary care among cancer survivors. $\mathrm{Br} \mathrm{J}$ Cancer 105(Suppl 1): S46-S51
Khan NF, Mant D, Carpenter L, Forman D, Rose PW (2011a) Long-term health outcomes in a British cohort of breast, colorectal and prostate cancer survivors: a database study. Br J Cancer 105(Suppl 1): S29-S37

Maddams J, Brewster D, Gavin A, Steward J, Elliott J, Moller H (2009) Cancer prevalence in the United Kingdom: estimates for 2008. Br J Cancer 101(3): $541-547$

Maddams J, Utley M, Møller H (2011a) A person-time analysis of hospital activity among cancer survivors in England. Br J Cancer 105(Suppl 1): $\mathrm{S} 38-\mathrm{S} 45$

Maddams J, Utley M, Moller H (2011b) Levels of acute service use among cancer survivors in the United Kingdom. Eur J Cancer 47(14): 2211 - 2220

Maher J, McConnell H (2011) New pathways of care for cancer survivors: adding the numbers. Br J Cancer 105(Suppl 1): S5-S10

NCRI (2004) Supportive and Palliative Care Research in the UK: Report of the NCRI Strategic Planning Group on Supportive and Palliative Care. http://www.ncri.org.uk/includes/Publications/reports/ncri_supportive and_palliative_report_web.pdf

NCRI (2010) Rapid Review of Research in Survivorship After Cancer and End of Life Care. National Cancer Research Institute: London

Richardson A, Addington-Hall J, Amir Z, Foster C, Stark D, Armes J, Brearley SG, Hodges L, Hook J, Jarret N, Stamataki Z, Scott I, Walker J, Ziegler L, Sharpe M (2011) Knowledge, ignorance and priorities for research in key areas of cancer survivorship: findings from a scoping review. Br J Cancer 105(Suppl 1): S82-S94

Tritter JQ, Calnan M (2002) Cancer as a chronic illness? Recondsidering categorization and exploring experience. Eur J Cancer Care 11(3): $161-165$

cc)(-) This work is licensed under the Creative Commons BY NC SA Attribution-NonCommercial-Share Alike 3.0 Unported License. To view a copy of this license, visit http://creativecommons. org/licenses/by-nc-sa/3.0/ 